\title{
Tunneling Transistor by Quantum Anomalous Spin Hall Effect of Phase Isolator
}

\author{
Received October 5, 2020; revised October 30, 2020; accepted November 1, 2020
}

\author{
Xiangjiang $\mathrm{Li}^{\mathrm{a}}$ and Teresa $\mathrm{Oh}^{\mathrm{b}, *}$ \\ aschool of Information Engineering, Nanyang Institute of Technology, Nanyang 473004, China \\ ${ }^{b}$ Department of Semiconductor Engineering, Cheongju University, Cheongju 28503, Republic of Korea
}

*Corresponding author E-mail: teresa@cju.ac.kr

\begin{abstract}
We found that transistors consisting of the gate insulation film exhibit bi-directional transfer characteristics, and when the gate voltage varies from negative to positive, drain current represents the values of positive and negative current, respectively. If the gate voltage is $0 \mathrm{~V}$, it is confirmed that tunneling occurs and the resistance is zero. The drain voltage is low, the resistance is reduced. As the drain voltage increases, the resistance increases. We observed a shift in the $\mathrm{I}_{\mathrm{DS}}-\mathrm{V}_{\mathrm{GS}}$ curve. The current generated through the gate insulation is the surface current caused by the antimatters, and quantum anomalous Hall effect of $\mathrm{I}_{\mathrm{DS}}-\mathrm{V}_{\mathrm{GS}}$ transmission characteristics was observed even in the absence of applied magnetic field. It is shown that $\mathrm{R}=0$ is a phase-isolator with excellent insulation properties of the SiOC thin film. The reason for the increased conductivity of the $100{ }^{\circ} \mathrm{C}$ heat-treated thin film is due to the spin current, and the thin films at other temperature is due to the surface current.
\end{abstract}

Keywords: Phase isolator, SiOC, Magnetoresistance, Quantum anomalous Hall effect

\section{Introduction}

Electromagnetic energy is generally applicable in nearly all electronic devices regardless of its size of scale down. Smaller sizes face technological limitations, where commonly known physical phenomena are not observed. The physical world can be understood through classical physics and quantum physics, and particles and mass are the starting points for the general physical phenomenon in which physics begins [1-4]. The electron and spin are the same amount of energy, but the sources of energy are very different. The electron is a particle and comes from electrical energy, the spin is an importer and comes from magnetic energy. It is traditional physics that electrons move, and if electrons fail to move, they must be interpreted in quantum physics. This is consistent with the difference between omic and magnetic resistance. Particles have mass, and as a result, they follow laws of energy conservation. There is something that has the energy that is non massive antiparticle, but it is a photon (light). Light does not have mass but carries energy. To explain this, antiparticles were introduced. The spin current is not a particle but has energy because it has no mass. Light, photon, and proton are called antimatters, and because they can be interpreted mathematically by applying energy conservation laws only when they have mass, the mass of the antimatter was applied to the mass of electrons. As a result, matter analysis is general physics, and antimatter analysis is quantum physics. Classical physics cannot explain the behavior of very small particles and we need to employ quantum physics [5-8]. Electromagnetic resistance could be either positive or negative. Positive resistance can be described using ohmic resistance characteristics. However, nothing is known about negative resistance. Surface current consists of spin current and charge current. The source of the current is in electrons and spins, and the direction of electrons and spins in a two-dimensional structure is in the opposite direction because they originate from electricity and magnetism. The resistance of electrical energy is positive ohmic resistance. The resistance of magnetic energy is negative magnetic resistance. Magnetoresistance is a phenomenon caused by a Schottky contact and potential barrier. This study focuses on the electrical features that occur in the negative resistance regime [9-12].

Figure 1 shows the correlation between magnetic resistance and conductivity in phase isolators, that phase-isolators is materials with spin currents in spite of insulators. The top figure shows currentvoltage transfer characteristics as an electrical properties, and the bottom figure illustrates the relationship between magnetic resistance in an insulator and conductivity in a circle as fermi plane. If there is a positive resistance as shown in Figs. 1(a) and 1(b), the characteristics of n-type or p-type semiconductor characteristics are unipolar transfer characteristics above the threshold voltage. When resistance decreases, carriers tunnel, and when resistance increases, conductivity decreases. As shown in Figs. 1(c) and 1(d), the resistance becomes negative by the Schottky contact and the magnetoresistance appears. Quantum anomalous spin Hall effects explain the principle that magnetic resistance caused by a phenomenon in which spin current occurs without a magnetic field is made in a phase isolator. Smaller magnetoresistance results in higher conductivity and bi-directional transmission characteristics [13-15]. As the resistance becomes lower and lower, quantum tunneling by surface current occurs.

In this study, transistor using low-k (low dielectric constant materials) $\mathrm{SiOC}$ as gate isolators were manufactured to investigate the 

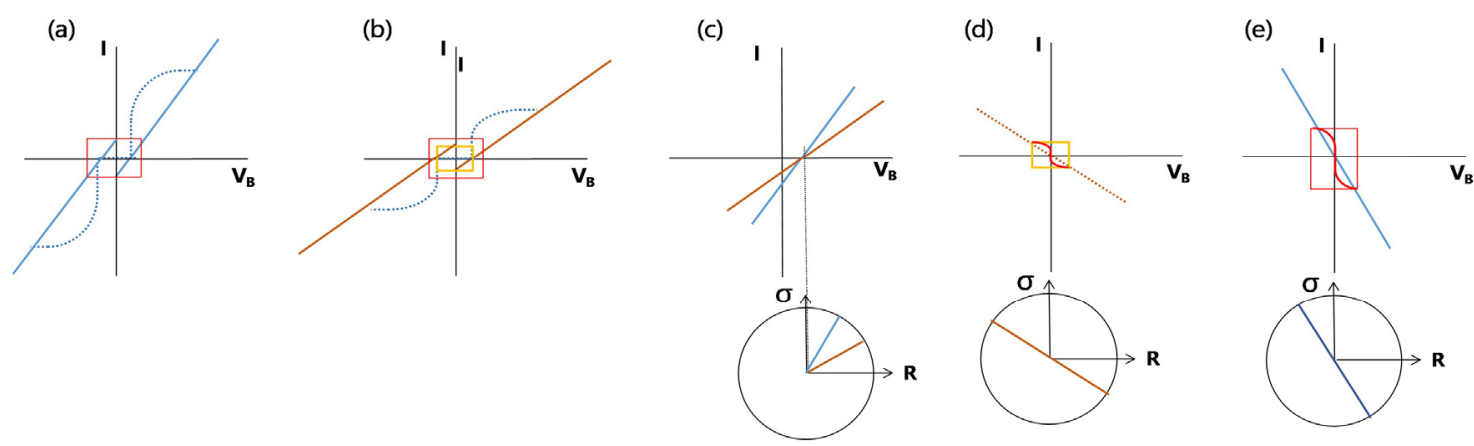

Figure 1. Correlation between conductivity and resistance, (a) (b) positive resistance, (c) (d) negative resistance, and (e) positive resistance.
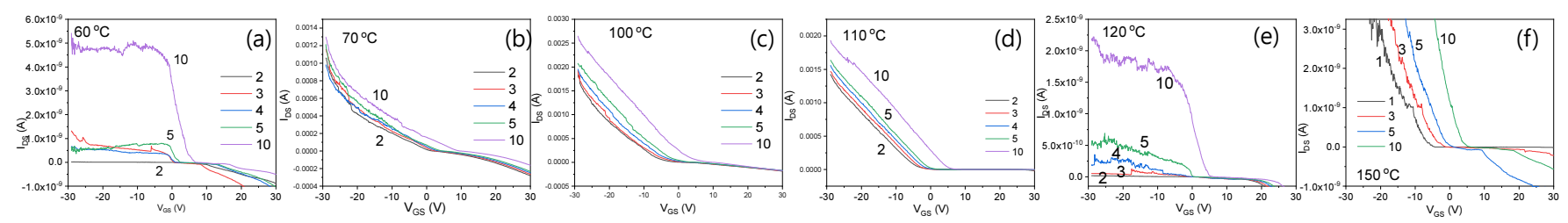

Figure 2. Transfer characteristics of $\mathrm{IDS}_{\mathrm{S}} \mathrm{V}_{\mathrm{GS}}$ of transistors with temperature variation in $2 \mathrm{~V} \leq \mathrm{V}_{D S} \leq 10 \mathrm{~V}$.
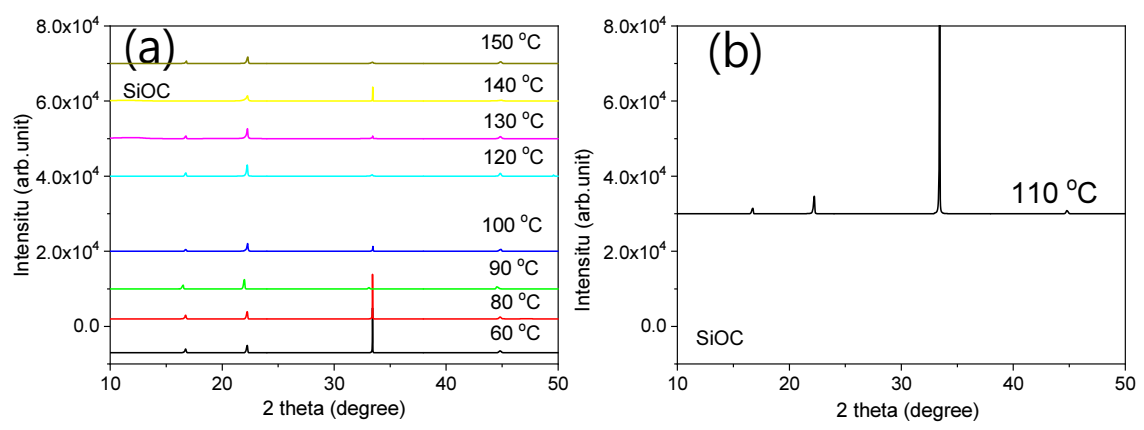

Figure 3. XRD patterns of $\mathrm{SiOC}$ films annealed at (a) $60 \sim 150{ }^{\circ} \mathrm{C}$ and (b) $110{ }^{\circ} \mathrm{C}$.

causes of the current generation without channels. We performed $\mathrm{X}$-ray diffraction (XRD) analysis to observe the phase transition in the SiOC thin film. The origin of surface current in phase-isolators and the causes of superior transfer characteristics in transistors using SiOC where phase transition occurs were investigated.

\section{Experimental details}

The SiOC as a gate insulator was prepared on the Si wafer by employing oxygen flow at the rate of $20 \mathrm{sccm}$ using magnetron sputtering system at $250 \mathrm{RF}$ power for $10 \mathrm{~min}$. The source-target was the SiOC fabricated from Nano Technology, Inc., Korea. The asdeposited SiOC films were annealed at various temperatures in a vacuum with 3 mTorr. To make the transistors, the MIS (metal/ insulator $/ \mathrm{Si}$ ) structured electrodes were made with aluminum to measure electrical properties using mask patterns of source, drain, and gate electrodes. The electrical characteristics of the device were measured using a semiconductor parameter analyzer (HP4155A).

\section{Results and discussion}

Figures 2(a)-(f) shows the transfer characteristics of $I_{D S}-V_{G S}$ as electrical properties of transistors due to temperature change in the region of $2 \mathrm{~V} \leq \mathrm{V}_{\mathrm{DS}} \leq 10 \mathrm{~V}$. Depending on the characteristics of the phase-isolator, the current is $+\mathrm{I}_{\mathrm{DS}}$ when it is $-\mathrm{V}_{\mathrm{GS}}$, and $-\mathrm{I}_{\mathrm{DS}}$ for $+\mathrm{V}_{\mathrm{GS}}$, indicating bi-directional transfer characteristics. A significant change of a sudden increase in current takes place between 110 and $120{ }^{\circ} \mathrm{C}$. The transfer characteristic at $110{ }^{\circ} \mathrm{C}$ was obtained because the electron polarization was the lowest at $110^{\circ} \mathrm{C}$ due to the crystallinity by the low electron polarization during the heat treatment of SiOC, and the plot at $120{ }^{\circ} \mathrm{C}$ showed that the transfer characteristic indicates an increase in the charge current, where the phenomenon of electron polarization is not found.

Figure 3(a) shows an XRD pattern showing the crystallization of SiOC thin films. Most of the SiOC thin films exhibit amorphous properties, while SiOC thin films that are annealed at $110{ }^{\circ} \mathrm{C}$ are highly crystallized [16]. This implies that the phase state changes only post the $110{ }^{\circ} \mathrm{C}$ annealing process. $\mathrm{I}_{\mathrm{DS}}-\mathrm{V}_{\mathrm{GS}}$ curves were investigated in detail to understand changes in the electrical properties upon phase change at $110{ }^{\circ} \mathrm{C}$.

Figure 4 shows the $\mathrm{I}_{\mathrm{DS}}-\mathrm{V}_{\mathrm{GS}}$ transfer characteristics of transistors according to temperature changes in the $0.001 \mathrm{~V} \leq \mathrm{V}_{\mathrm{DS}} \leq 1 \mathrm{~V}$ region to observe the junction characteristics of SiOC thin films with various annealing temperatures. The upper panels show the transfer charac- 


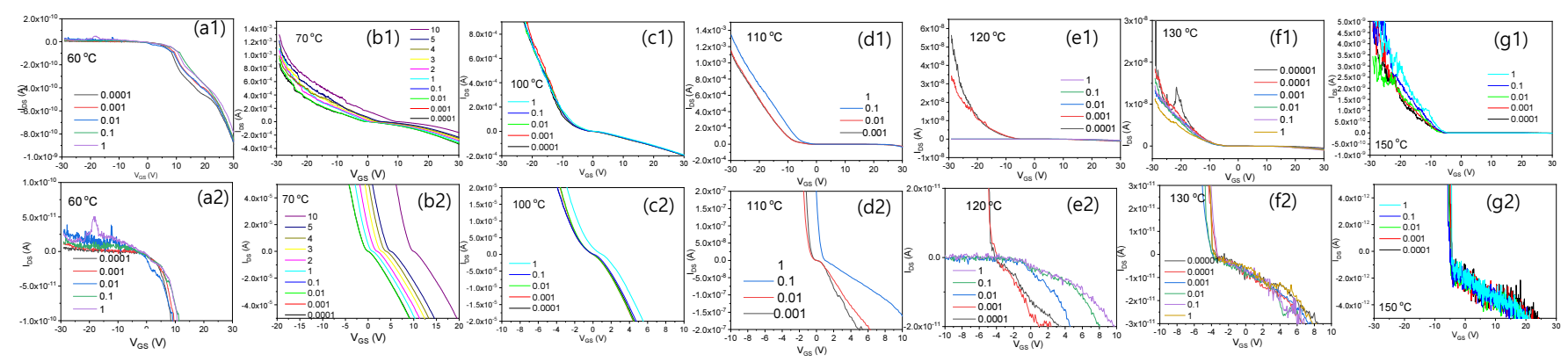

Figure 4. Transfer characteristics of $\mathrm{IDS}_{\mathrm{DS}} \mathrm{V}_{\mathrm{GS}}$ of transistors with temperature variation in the range $0.001 \mathrm{~V} \leq \mathrm{V}_{D S} \leq 1 \mathrm{~V}$, upper panels represent high current at micro scale and bottom panels represent low current at nano scale, (a) 60, (b) 70, (c) 100, (d) 110, (e) 120, (f) 130, and (g) $150{ }^{\circ} \mathrm{C}$.

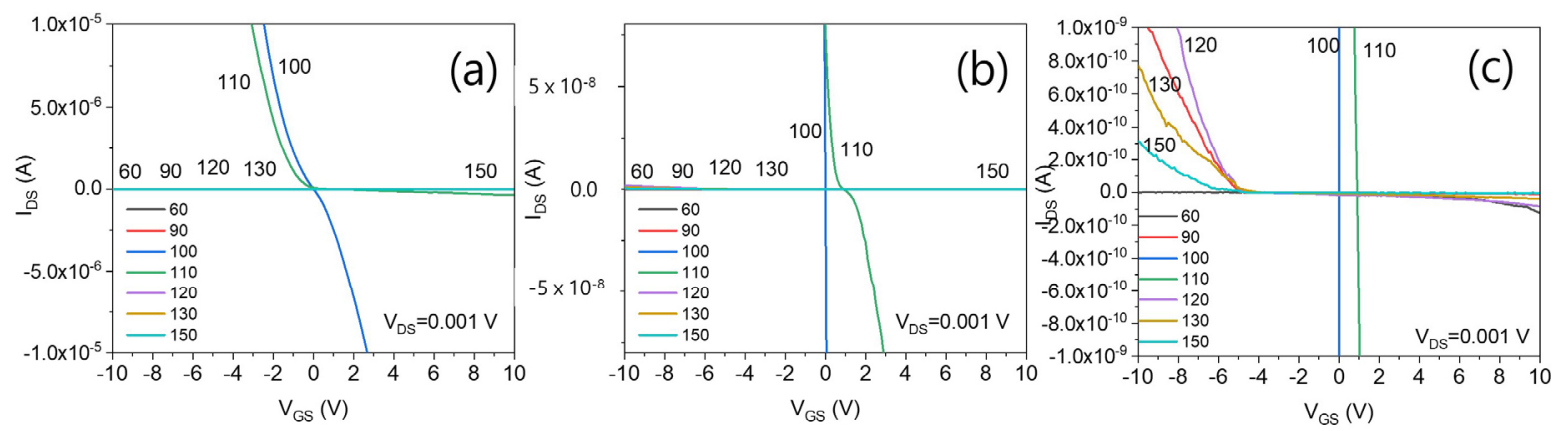

Figure 5. Transfer characteristics of $\mathrm{I}_{D S}-\mathrm{V}_{G S}$ of transistors with $\mathrm{V}_{D S}=0.001 \mathrm{~V}$, (a) higher $\mathrm{I}_{D S}$ at $100{ }^{\circ} \mathrm{C}$ annealed transistor than that at $110{ }^{\circ} \mathrm{C}$ annealed transistor, (b) Schottky contact at $110{ }^{\circ} \mathrm{C}$ annealed transistor, and (c) magnetic resistance characteristics of transistors annealed above $110{ }^{\circ} \mathrm{C}$.

teristics and the bottom panels represent the formation characteristics of Schottky contacts. Ohmic resistance is formed by the ohmic contact and negative resistance is formed by the Schottky contact. The characteristics of the Schottky contact can be investigated in Fig. 4 bottom panels. The characteristics of the Schottky contact can be seen from the shape of the current curve changing as the voltage changes from negative to positive. Positive (+) current and negative (-) current contacts can be seen well-formed in a thin film at $110{ }^{\circ} \mathrm{C}$ with symmetrical values of current. It can be seen that dual Schottky connectivity characteristics are seen in a $110{ }^{\circ} \mathrm{C}$ SiOC transistor. Shottky contacts are also shown in thin films that are annealed at 70 ${ }^{\circ} \mathrm{C}$ of Fig. 4(b2) and $100{ }^{\circ} \mathrm{C}$ of Fig. 4(c2). When the Schottky contact is formed in double formation, the transfer characteristics can achieve better electrical characteristics, such as low resistance and a high degree of movement.

Figure 5 compares the $\mathrm{I}_{\mathrm{DS}}-\mathrm{V}_{\mathrm{GS}}$ delivery characteristics at $\mathrm{V}_{\mathrm{DS}}=$ $0.001 \mathrm{~V}$. In the $\sim \mu \mathrm{A}$ regime, in Fig. 5(a), more current flows from the $100{ }^{\circ} \mathrm{C}$ heat-treated thin film. Figure 5(b) shows the lower current $\sim \times 10^{-8}$ A regime and the creation of a Schottky contact in a $110{ }^{\circ} \mathrm{C}$ heat-treated thin-film, and it can be seen that the tunneling phenomenon as bi-directional properties are more speedable at a $100{ }^{\circ} \mathrm{C}$ heat-treated thin film.

To understand the magnetic resistance characteristics, the transfer characteristics according to the heat treatment temperature in the $\sim \times 10^{-10}$ A regime are compared as shown in Fig. 5(c). At temperatures above $110{ }^{\circ} \mathrm{C}$, the current decreases with an increase in the annealing temperature.

Indicates a typical magnetic resistance characteristic in which the current decreases as the temperature rises. There is a correlation between magnetic resistance characteristics and the Schottky contact, and the Schottky contact was formed in a $110{ }^{\circ} \mathrm{C}$ heat-treated devices, and the transistor with the Schottky contact had electrically superior transfer characteristics.

Figure 6 investigated the transfer characteristics of $\mathrm{I}_{\mathrm{DS}}-\mathrm{V}_{\mathrm{GS}}$ to compare the $90{ }^{\circ} \mathrm{C}$ transistor and the $110{ }^{\circ} \mathrm{C}$ transistor with the Schottky contact. As shown in Figs. 6(a) and 6(b), $110{ }^{\circ} \mathrm{C}$ transistor had better $\mathrm{I}_{\mathrm{DS}}-\mathrm{V}_{\mathrm{GS}}$ transfer characteristics than that of $90{ }^{\circ} \mathrm{C}$ heat treated units. Figure 6(c) compared the $\mathrm{I}_{\mathrm{DS}}-\mathrm{V}_{\mathrm{GS}}$ characteristics of 100 and $110{ }^{\circ} \mathrm{C}$ elements by plotting them on a log scale. A $100{ }^{\circ} \mathrm{C}$ annealed device with a tunneling effect exhibited excellent transmission characteristics.

Figure 7 represents the logarithmic plots of the transfer characteristics of $\mathrm{I}_{\mathrm{DS}}-\mathrm{V}_{\mathrm{GS}}$ measured in the $0.0001 \mathrm{~V} \leq \mathrm{V}_{\mathrm{DS}} \leq 1 \mathrm{~V}$ region to investigate the switching characteristics of the transistors. Compared to the transmission characteristics of the $100{ }^{\circ} \mathrm{C}$ annealed transistor's $\mathrm{V}_{\mathrm{DS}}=1 \mathrm{~V}$, it looks different. For further investigation, only the $\mathrm{I}_{\mathrm{DS}}$ curves for $\mathrm{V}_{\mathrm{DS}}=1 \mathrm{~V}$ were classified and compared as shown in Fig. 8.

Figure 8 shows the logarithmic plots of heat-treated transistors above $90{ }^{\circ} \mathrm{C}$ at $\mathrm{V}_{\mathrm{DS}}=1 \mathrm{~V}$. The switching characteristics of the transistors treated at $100{ }^{\circ} \mathrm{C}$, forming the Schottky contact were found to be the best. At temperatures beyond $100{ }^{\circ} \mathrm{C}$, the $\mathrm{I}_{\mathrm{DS}}$ current showed a decreasing magnetic resistance characteristic. The magnetic resistance characteristic of phase-isolator implies a quantum anomalous Hall phenomenon, which is a phenomenon where current flows even without magnetic fields. The quantum hole effect has a singularity of supercurrent. XRD and Shocky Joint were used as a way to find out the uniqueness of super current, and it was shown that super current was flowing in a $100{ }^{\circ} \mathrm{C}$ heat-treated thin film with relatively high current flow. The bi-directional transfer characteristic of transistors is a quantum tunneling phenomenon. Tunneling phenomena include electronic tunneling and quantum tunneling. Electronic tunneling by 

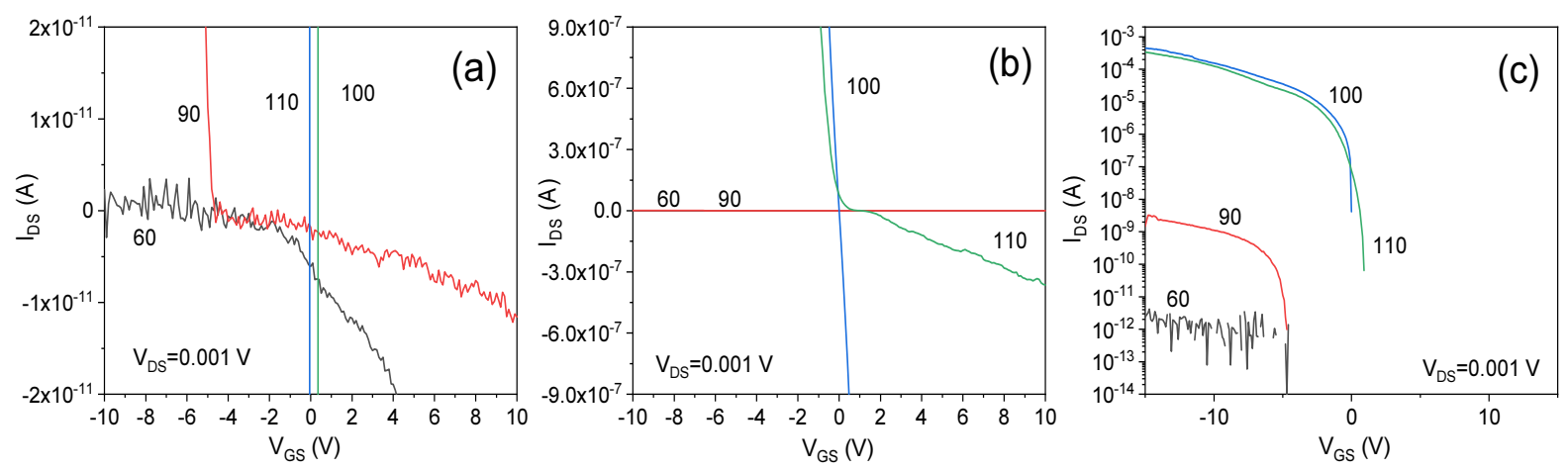

Figure 6. Analysis of transistors with Schottky contacts, (a) $90{ }^{\circ} \mathrm{C}$ annealed transistor with Schottky contact, (b) $110{ }^{\circ} \mathrm{C}$ annealed transistor with Schottky contact, and (c) IDS comparison between 100 and $110{ }^{\circ} \mathrm{C}$ annealed transistors.
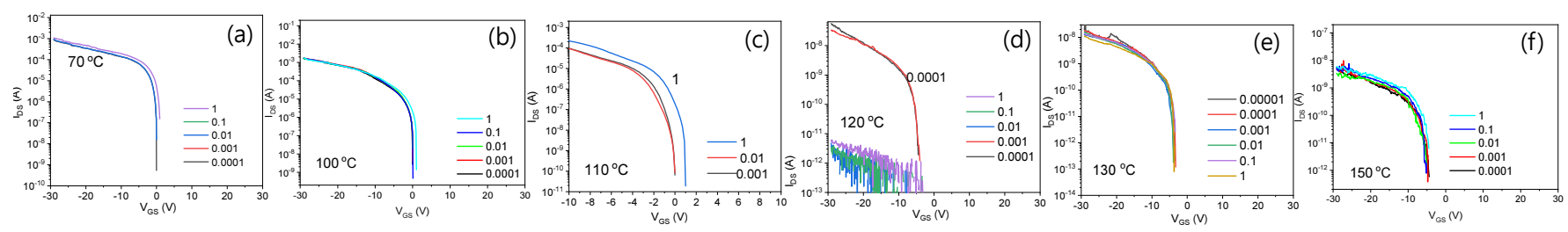

Figure 7. Log transformation of the transfer characteristics of $\mathrm{IDS}_{\mathrm{S}} \mathrm{V}_{\mathrm{GS}}$, (a) 70 , (b) 100, (c) 110, (d) 120 , (e) 130 , and (f) $150{ }^{\circ} \mathrm{C}$.
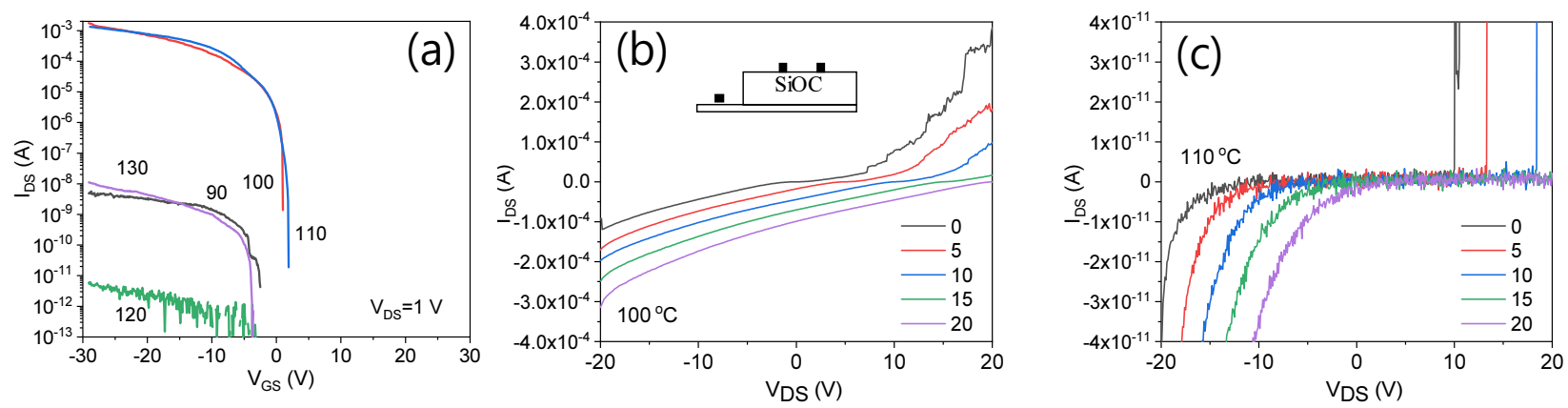

Figure 8. Stability of transistors, (a) log transformation of transfer characteristics of $\mathrm{I}_{D S}-\mathrm{V}_{G S}$ at $\mathrm{V}_{D S}=1 \mathrm{~V}$ of transistors annealed above $90{ }^{\circ} \mathrm{C}$, (b) and (c) comparison of $S c h o t t k y$ contacts of $\mathrm{I}_{D S}-\mathrm{V}_{D S}$.

high concentration doping does not have magnetic resistance characteristics, indicating one-way transfer characteristics. Quantum tunneling, however, showed bi-directional transfer characteristics as it has magnetic resistance characteristics. A phase insulator with magnetic resistance due to a potential barrier has a spin current flowing only on the surface [10]. Surface current is characteristic of quantum anomalous Hall phenomena. Here, the quantum anomalous Hall phenomena and quantum tunneling phenomena are the same. The properties of transistors using the phase-isolated $\mathrm{SiOC}$ thin film were superior in the case of Schottky contacts.

The reason for observing bi-directional transfer characteristics in transistors that use gate insulation membrane without channels is the flow of the surface current of phase isolation. As the characteristics of the phase-isolator allow for magnetic resistance, and in thin-film transistors, the gate insulation has magnetic resistance, the electrical efficiency of SiOC thin film where phase transition occurs was observed to increase. The tunneling effect or superconducting phenomenon occurs where resistance is reduced. Resistance and conductivity are inversely related to each other, and positive and negative resistance are symmetrical to each other.

\section{Conclusions}

Low polarity SiOC thin film is known as low-k material with good insulation. Thin film transistors with bi-directional transfer characteristics were created using low-k SiOC with gate insulation, but channels were not used. The reason why transistors operate is because low-k SiOC has phase-insulated properties and thus, surface current flows. In particular, we observed that the switching characteristics of transistors in SiOC thin films with $110{ }^{\circ} \mathrm{C}$ heat treatment were better. The XRD analysis of SiOC thin film showed that spin current occurs where the Schottky connection is the best. This is because XRD can be used as a way to find out the specificity of thin films that meet the conditions of super current flow.

\section{References}

[1] M. Sakr and S. Liu, Renew. Sust. Energ. Rev. 39, 262 (2014). 
[2] Z. Fan, S. N. Mohammad, W. Kim, Ö. Aktas, A. E. Botchkarev, and H. Morkoç, Appl. Phys. Lett. 68, 1672 (1996).

[3] C. L. Kane, Nat. Phys. 4, 348 (2008).

[4] J. Maserjian and N. Zamani, Appl. Phys. Lett. 53, 559 (1982).

[5] N. M. Ravindra and J. Zhao, Smart Mater. Struct. 1, 197 (1992).

[6] H. L. Mosbacker, Y. M. Strzhemechny, B. D. White, P. E. Smith, D. C. Look, D. C. Reynolds, C. W. Litton, and L. J. Brillson, Appl. Phys. Lett. 87, 012102 (2015).

[7] D. Yang, F. Wang, Y. Ren, Y. Zuo, Y. Peng, S. Zhou, and D. Xue, Adv. Funct. Mater. 23, 2918 (2013).

[8] Y. Suzuki and H. Kubota, J. Phys. Soc. Jpn. 77, 031002 (2008).

[9] T. Oh, J. Nanosci. Nanotechnol. 19, 2174 (2019).
[10] T. Oh, Sci. Rep. 10, 9509 (2020).

[11] E. G. Barbagiovanni, D. J. Lockwood, P. J. Simpson, and L. V. Goncharova, Appl. Phys. Rev. 1, 011302 (2014).

[12] T. Oh, J. Nanosci. Nanotechnol. 17, 7470 (2017).

[13] O. Mitrofanov and Michael Mantra, J. Appl. Phys. 95, 6414 (2004).

[14] M. P. Delmo, S. Yamamoto, S. Kasai, T. Ono, and K. Kobayashi, Nature 457, 1112 (2009).

[15] N. V. Volkov, A. S. Tarasov, E. V. Eremin, F. A. Baron, S. N. Varnakov, and S. G. Ovchinnikov, J. Appl. Phys. 114, 093903 (2013).

[16] T. Oh, K. S. Oh, K. M. Lee, and C. K. Choi, Thin Solid Films 468, 316 (2004). 